\title{
VERIFICAÇÃO DAS HABILIDADES FUNCIONAIS E NECESSIDADES DE AUXÍLIO DO CUIDADOR EM CRIANÇAS COM PARALISIA CEREBRAL NOS PRIMEIROS MESES DE VIDA
}

\author{
VERIFICATION OF THE FUNCTIONAL ABILITIES AND ASSISTANCE \\ NEEDS OF CHILDREN WITH CEREBRAL PALSY
}

Herrero D, Monteiro CBM. Verificação das Habilidades Funcionais e Necessidades de Auxílio do Cuidador em Crianças com Paralisia Cerebral nos Primeiros Meses de Vida. Rev Bras Crescimento Desenvol Hum. 2008; 18(2): 163-169.

Resumo: Introdução: Paralisia Cerebral é uma desordem do movimento e da postura, persistente, porém variável, surgida nos primeiros anos de vida pela interferência no desenvolvimento do sistema nervoso central, causada por uma desordem cerebral não progressiva ${ }^{1}$. Tem como principal característica a inabilidade do corpo em enfrentar com eficiência os efeitos da gravidade. Devido às alterações motoras é importante a participação do Fisioterapeuta na equipe interdisciplinar. Para tanto, deve-se realizar avaliações das dificuldades na paralisia cerebral para possibilitar uma melhor organização do programa fisioterapêutico. Objetivo: Avaliar as habilidades funcionais e a necessidade de auxílio do cuidador em crianças com até 18 meses de idade, e com paralisia cerebral. Método: Foram avaliadas 7 crianças com diagnóstico médico de paralisia cerebral, entre 10 e 18 meses de idade, com média de idade correspondente a um ano e dois meses. A avaliação ocorreu por meio do P.E.D.I. e foram analisados os escores bruto e normativo nas áreas de habilidades funcionais e assistência do cuidador. Resultados: Verificou-se maior dificuldade nas crianças com paralisia cerebral na área de mobilidade e, desta forma, a necessidade de maior assistência em movimentos e posturas. Conclusão: Apesar das alterações nos componentes neuromotores observadas em crianças de risco, as mesmas acabam desenvolvendo estratégias motoras que lhes permitem desempenhar atividades funcionais, como por exemplo as situações citadas na área de auto-cuidado. Os cuidadores orientados pelo fisioterapeuta podem propor facilitações que beneficiarão a função da criança, sua participação na atividade e desenvolvimento da comunicação.

Palavras-chave: Paralisia cerebral; desenvolvimento infantil; fisioterapia; intervenção precoce; função; avaliação.

\section{INTRODUÇ̃̃O}

Bobath $^{2}$ complementa a definição como sendo “...devido a fatores hereditários, eventos ocorridos durante a gravidez, parto, período neonatal ou durante os primeiros dois anos de vida".

Conforme se observa na definição acima, é necessário e fundamental que o paciente apre-

\footnotetext{
Fisioterapeuta Especialista em Pediatria pela Universidade Cidade de São Paulo (UNICID). Endereço: R. Cesário Galeno, 448/475 - Tatuapé - São Paulo - SP. E-mail: dafneh@uol.com.br

** Fisioterapeuta. Doutor. Professor de Pediatria da Faculdades Metropolitanas Unidas (FMU). Endereço: Avenida Santo Amaro,1239- Vila Nova Conceição - São Paulo - SP.

Trabalho de conclusão do curso de pós-graduação para obtenção do título de especialista em Fisioterapia Pediátrica pela UNICID.

Instituição de Realização do Trabalho: Centro Israelita de Apoio Multidisciplinar (CIAM), R. Irmã Pia, 78 - Jaguaré - São Paulo - SP.
} 
sente uma desordem ou distúrbio da postura e do movimento para ter o diagnóstico médico de Paralisia Cerebral (PC), sendo que os distúrbios de postura e movimento podem ser definidos como a inabilidade do corpo em enfrentar com eficiência os efeitos da gravidade, e de se relacionar com a superfície da terra por meio da base de apoio. ${ }^{3}$

As dificuldades em manter uma postura e realizar um movimento são devidas, principalmente, as alterações de tônus e a presença de padrões anormais de movimento. Tal fato não permite a aquisição de reações posturais normais e, desta forma, a criança não consegue manter seu alinhamento e retificação para vivenciar suas atividades diárias e se manter contra gravidade em diversas posturas. ${ }^{4}$ Isso implica em perda de funcionalidade, dependência na maioria de suas atividades do cotidiano e necessidade de sua inclusão em programas de reabilitação contínuos. ${ }^{5}$

Devido ao envolvimento de alterações da postura e movimento, é importante para a criança com PC a participação do fisioterapeuta na equipe de habilitação. No entanto, é fundamental para os fisioterapeutas conhecer as características da doença e as necessidades do paciente para viabilizar um tratamento de acordo com as suas carências.

As aquisições motoras nos primeiros anos de vida são fatores relevantes no prognóstico do desenvolvimento global da criança, pois o período compreendido entre o nascimento e o final do primeiro ano de vida é considerado como um dos mais críticos no desenvolvimento infantil ${ }^{6}$. Neste período, o desenvolvimento motor apresenta ritmo acelerado de mudanças que culminam nas funções de mobilidade, com a aquisição do engatinhar e da marcha independente, respectivamente aos 9 e 12 meses de idade. ${ }^{6}$ A criança que apresenta paralisia cerebral pode apresentar atraso nos marcos de desenvolvimento. ${ }^{5}$

Tais atrasos podem ser investigados em seu cotidiano através do instrumento de avaliação P.E.D.I., questionário feito com cuidadores ou terapeutas que tem por objetivo identificar desenvolvimento típico de cada criança de acordo com algumas áreas de habilidades funcionais, assistência do cuidador e modificações. As áreas destacadas no estudo serão de habilidades funcionais e assistência do cuidador.

Este estudo transversal tem por objetivo investigar as habilidades funcionais e a necessidade de auxílio de portadores de PC que freqüentam um centro de reabilitação especializado. Essa informação poderá contribuir para um melhor acompanhamento em longo prazo desses pacientes, assim como direcionar o fisioterapeuta para minimizar as maiores dificuldades das crianças.

\section{OBJETIVO}

Avaliar as habilidades funcionais e a necessidade de auxílio do cuidador em crianças com paralisia cerebral até 18 meses de idade.

\section{MÉTODO}

Para a realização deste trabalho foram avaliadas 7 crianças, quatro do sexo feminino e três do sexo masculino, com diagnóstico médico de PC, com idade, por ocasião da investigação, que variou entre 10 e 18 meses, com média de 14 meses. O quadro motor apresentado por três crianças era de diparesia, pela aquisição do sentar mostrada por volta dos 14 meses de idade, e o restante das crianças apresentava tetraparesia.

A avaliação das habilidades funcionais e da necessidade de assistência por parte do cuidador foi realizada utilizando-se o Inventário de Avaliação Pediátrica de Incapacidade, P.E.D.I. (Pediatric Evaluation of Disability Inventory), desenvolvido por Haley et al. ${ }^{8}$ e validado para a população brasileira por Mancini. ${ }^{7}$ Esse instrumento de avaliação tem como característica principal coletar informações a respeito da capacidade e desempenho em três áreas de atividades: habilidades funcionais (consiste em aquisições da criança nas áreas de autocuidado, mobilidade e função social), assistência do cuidador (são repetidas as sub-áreas citadas anteriormente, mas avalia o quanto de ajuda a criança recebe do cuidador para realizar a atividade) e modificações (corresponde às adaptações necessárias para que a crianças realize as atividades, por 
exemplo uso de órteses, próteses ou outro tipo de adaptação no ambiente sendo específica para criança com deficiência ou modificação geral para qualquer criança, por exemplo o uso de engrossador na colher para criança com deficiência e o uso de redutor de vaso para qualquer criança). Esta última parte foi descartada no estudo pelo fato das crianças estarem se adaptando ao uso das goteiras, recentemente adquiridas, e as modificações no ambiente doméstico estarem começando a acontecer.

Para a averiguação das habilidades funcionais, foram avaliadas 73 atividades de autocuidado, 59 de mobilidade e 65 de função social, perfazendo um total de 197 atividades nas quais cada paciente foi considerado capaz, pontuando 1 , ou incapaz, não pontuando. Para a determinação da necessidade de assistência por parte do cuidador, foram consideradas oito subáreas do autocuidado, sete da mobilidade e cinco da função social, sendo o grau de dependência caracterizado como sendo total (pontuação $=0$, a criança não participa ou antecipa a atividade), máximo (1, a criança reconhece o ambiente e mostra que identifica a situação), moderado (2, participa com alguma vocalização ou movimentação), mínimo (3, realiza movimentação com auxílio em pontos chave, por exemplo), necessita apenas supervisão (4, é necessário que tenha algum cuidador presente que o relembre de alguns passos na tarefa, por exemplo) e é independente $(5$, realiza sem a necessidade da atividade ser refeita por um adulto).

Após a aplicação do questionário obtevese 2 escores: bruto e normativo. O escore bruto (EB) indica o número que a criança pontuou nas perguntas respondidas pelos cuidadores. Já, o escore normativo (EN) indica o escore atingido quando comparadas às crianças de mesma faixa etária com desenvolvimento normal, e deve apresentar-se entre 30 e 70 . Aquelas que apresentaram escore inferior a 30 ilustram atraso ou desenvolvimento significativamente inferior ao demonstrado por crianças de mesma faixa etária. ${ }^{7}$ O P.E.DI. oferece ainda o escore contínuo que corresponde à pontuação de cada paciente comparada a um grupo de crianças de até 7 anos de idade. Neste caso o escore foi descartado na análise pelo fato da idade das crianças no estudo ser inferior a dois anos e não haver justificativa para compará-las com crianças mais velhas.

Para a aplicação do P.E.D.I., foi realizado o treinamento mínimo recomendado pelos autores ${ }^{7}$.E utilizou-se o método de entrevista simultânea à observação direta: os cuidadores respondem às perguntas e o terapeuta observa as reações e aquisições apresentadas pela criança.

O trabalho foi avaliado pelo comitê de ética da Universidade Cidade de São Paulo (incluir o número do protocolo), que exigiu a assinatura dos responsáveis pelas crianças em um termo de consentimento livre e esclarecido, se disponibilizando a participar da pesquisa, porém com liberdade para desistir sem que o atendimento sofresse algum prejuízo.

\section{RESULTADO}

A seguir apresentar-se-ão os resultados por meio de tabelas. A tabela 1 contém a distribuição do grupo de lactentes avaliado considerando o sexo e idade em meses: observa-se que foram avaliadas sete pacientes (quatro meninas e três meninos), idades entre 10 meses e 18 meses. A variação da idade gestacional está entre 28 e 37 semanas, com média de 32 semanas.

Tabela 1. Distribuição dos lactentes segundo sexo e idade em meses.

\begin{tabular}{lllllll}
\hline Idade em meses & \multicolumn{2}{c}{ Masculino } & \multicolumn{2}{c}{ Feminino } & \multicolumn{2}{c}{ Total } \\
\hline \multirow{2}{*}{$\begin{array}{l}* \\
10\end{array}$} & $\mathrm{n}$ & $\%$ & $\mathrm{~N}$ & $\%$ & $\mathrm{~N}$ & $\%$ \\
12 & 0 & 1 & 25 & 1 & 14 \\
17 & 1 & 33,33 & 0 & 0 & 1 & 14 \\
18 & 1 & 33,33 & 0 & 0 & 1 & 14 \\
Total & 1 & 33,33 & 3 & 75 & 4 & 58 \\
\hline
\end{tabular}


A tabela 2 apresenta os resultados individuais referentes às habilidades funcionais nas áreas de autocuidado, mobilidade e função social, informando os valores de escore bruto (soma total dos resultados) e normativo (valor comparativo com resultados de crianças da mesma fai- xa etária). Os resultados mostram que, com exceção do paciente número 4 , todos obtiveram escore abaixo do esperado para idade $(<30)$ apenas em mobilidade. Acrescenta-se que o quadro motor do paciente 4 é de tetraparesia por seqüela de meningite.

Tabela 2. Distribuição dos lactentes segundo escore obtido pelo P.E.D.I. na área de habilidades funcionais.

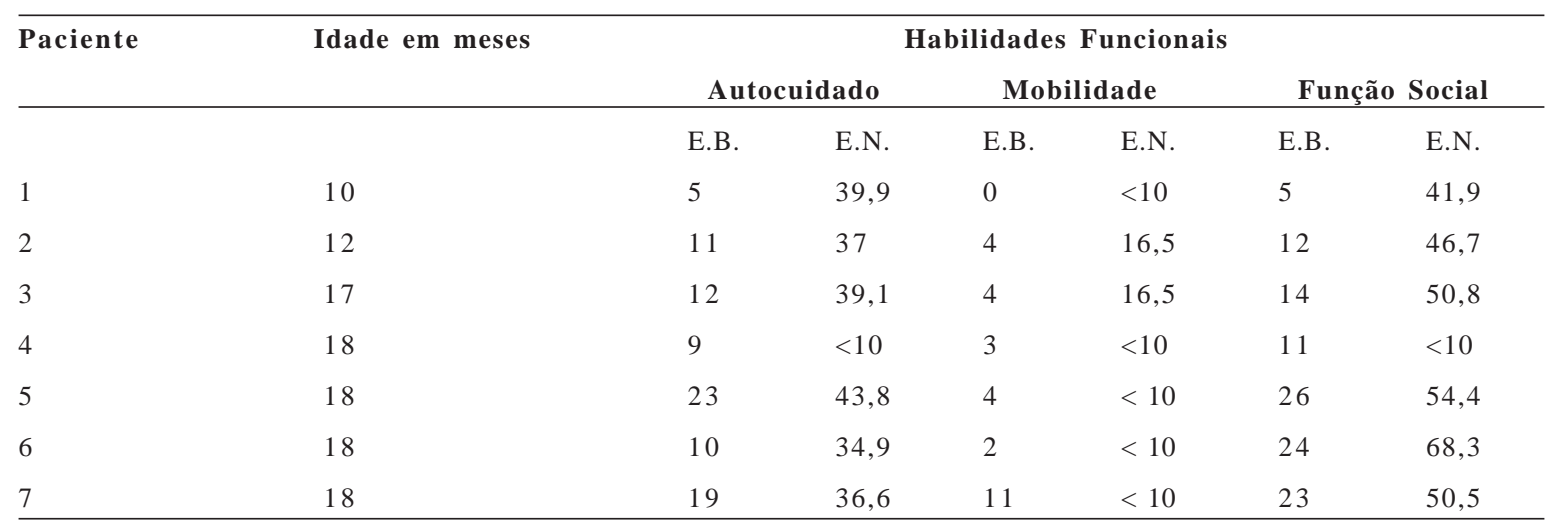

Legenda: E.B. : escore bruto; E.N. : escore normativo.

A tabela 3 apresenta os resultados individuais referentes à necessidade de assistência por parte do cuidador nas áreas de autocuidado, mobilidade e função social. Assim como nas habilidades funcionais acompanha informações dos valores de escore bruto (soma total dos resultados) e normativo (valor comparativo com resultados de crianças da mes- ma faixa etária). Os valores devem também estar entre 30 e 70 . Os relatos dos cuidadores devem identificar a participação da criança na atividade. Neste caso algo que poderia diminuir o escore das crianças é a realização das atividades sem que haja a percepção ou a oportunidade dada pelos cuidadores à participação ou não da criança na tarefa.

Tabela 3. Distribuição dos lactentes segundo escore obtido pelo P.E.D.I. na área de assistência do cuidador.

\begin{tabular}{|c|c|c|c|c|c|c|c|}
\hline \multirow[t]{3}{*}{ Paciente } & \multirow[t]{3}{*}{ Idade em meses } & \multicolumn{6}{|c|}{ Assistência do Cuidador } \\
\hline & & \multicolumn{2}{|c|}{ Auto Cuidado } & \multicolumn{2}{|c|}{ Mobilidade } & \multicolumn{2}{|c|}{ Função Social } \\
\hline & & E.B. & E.N. & E.B. & E.N. & E.B. & E.N. \\
\hline 1 & 10 & 0 & 37,2 & 0 & 32,8 & 0 & 39,2 \\
\hline 2 & 12 & 0 & 29,7 & 0 & $<10$ & 1 & 18,8 \\
\hline 4 & 18 & 3 & 31,5 & 0 & $<10$ & 3 & 30,1 \\
\hline 5 & 18 & 5 & 40,9 & 1 & $<10$ & 12 & 50,8 \\
\hline 6 & 18 & 2 & 37,3 & 1 & $<10$ & 18 & 73,4 \\
\hline 7 & 18 & 7 & 48,5 & 9 & 24,3 & 10 & 47,1 \\
\hline
\end{tabular}

Legenda: E.B. : escore bruto; E.N. : escore normativo. 


\section{DISCUSSÃO}

Para facilitar a compreensão da discussão optou-se em dividi-la em duas partes: 1- comparação entre as áreas de habilidades funcionais e 2- comparação entre as áreas de assistência do cuidador.

1- Comparação entre as áreas de habilidades funcionais.

Verifica-se que a área onde os pacientes obtiveram escore normativo menor foi na mobilidade, onde 5 pacientes $(71,5 \%)$ obtiveram valores abaixo de 10; enquanto que apenas 2 pacientes $(28,5 \%)$ apresentaram valor de 16,5 de escore normativo. Desta forma, todos os pacientes obtiveram resultados bastante abaixo do normal na área de mobilidade. Nota-se que a paciente 4 , do sexo feminino, apresenta todos os escores abaixo de 10, ou seja, tem um maior atraso do desenvolvimento em todas as áreas. Ressalta-se que o quadro motor em questão é de tetraparesia por sequiela de meningite.

Os outros pacientes estão dentro da faixa de normalidade $(30$ - 70) nas áreas de autocuidado e função social. Este resultado faz sentido, pois na paralisia cerebral a área de habilidade funcional que apresenta maior dificuldade é a mobilidade. Isto é verificado ao comparar com outras referências. Allegretti et al..$^{8}$ utilizaram o P.E.D.I. para investigar 10 crianças portadoras de PC, forma diparética espástica, com idade entre 4 e 5 anos, que foram comparadas com 10 crianças normais na mesma faixa etária. Os resultados mostraram diferença significativa entre as áreas de autocuidado e mobilidade, sendo que na mobilidade observou-se menos funcionalidade, ambas com $p<0,0001$. A função social não apresentou diferença significativa $(\mathrm{p}=0,132)$. Investigação realizada por Oliveira e Cordani ${ }^{9}$, que da mesma forma avaliaram crianças com PC do tipo diparética espástica, também detectou pior desempenho em mobilidade e melhor em função social.

Em outro estudo observou-se que o desenvolvimento de atividades funcionais de autocuidado também pode ser influenciado pela presença de PC. Tais resultados podem subsidiar estratégias de avaliação e intervenção para cri- anças com distúrbios neuromotores. ${ }^{5}$ Visto que para avaliação de autocuidado é necessário a aquisição de mobilidade funcional, novamente é ressaltada a dificuldade das crianças com PC em adaptar-se contra a gravidade e assumir posturas para realização das tarefas.

2- Comparação entre as áreas de assistência do cuidador

Quando se verifica o resultado da assistência do cuidador, percebe-se que a área da mobilidade necessita de maior auxílio por parte do cuidador, o que é bastante coerente com os resultados das habilidades funcionais, ou seja, a área que apresenta maior dificuldade necessita de maior auxílio.

Neste trabalho encontraram-se resultados para as três situações a seguir referentes à área da mobilidade, comparando-se as Tabelas 2 e 3 : a) o valor da assistência do cuidador é maior do que das habilidades funcionais (pacientes 1 e 7), o que significa que o cuidador auxilia a criança menos do que o necessário e hipervaloriza a capacidade do paciente; b) o valor da assistência do cuidador é menor do que das habilidades funcionais (pacientes 2 e 3 ), o que significa que o cuidador auxilia a criança mais do que o necessário e subvaloriza a capacidade do paciente; c) o valor da assistência do cuidador é igual ao das habilidades funcionais (pacientes 4,5 e 6 ), o que significa que o cuidador oferece o auxílio adequado para as limitações do paciente.

Considerando o autocuidado e a função social na assistência do cuidador, a grande maioria dos pacientes apresenta valores perto da normalidade (exceção para o paciente 2 em função social e 3 em autocuidado). O que novamente enfatiza que o auxílio na PC deve ser justamente na área de mobilidade.

Os resultados obtidos mostram a dificuldade apresentada pelas crianças portadoras de PC na área de mobilidade tanto nas habilidades funcionais, como no auxílio do cuidador. Desta forma, é importante o tratamento fisioterapêutico para propiciar melhoras funcionais ao paciente e uma maior participação no dia-a-dia.

A movimentação, segundo Hunsinger, ${ }^{10}$ proporciona à criança diferentes sensações (motoras, orgânicas, sensoriais, afetivas). Esta per- 
cebe, reproduz, revive e experimenta. Por isso se dá tamanha importância a um manuseio adequado em casa e durante a terapia, pois ao realizar a atividade com ajuda, a criança está aprendendo com o toque e a sensação. Se este é feito de forma adequada, a criança o reproduz de forma coerente e funcional e, principalmente, o memoriza adequadamente. Segundo Finnie ${ }^{11}$, a mobilidade pode ocorrer de forma independente, totalmente livre ou com um auxílio. Os pontos importantes na escolha de um auxílio que irá permitir que a criança seja móvel são: aumento de potencial para exploração do ambiente com segurança, desafio da sua capacidade de melhorar a independência e o não aumento do tônus anormal ou padrões pobres.

Devido ao P.E.D.I. ser um protocolo funcional, ele permite que durante sua aplicação sejam feitas orientações na rotina familiar. As adaptações são, na maioria das vezes, difíceis de serem aceitas. Porém, se ocorrerem de forma gradativa, a probabilidade de serem ouvidas, e principalmente seguidas de forma adequada, é grande.

Uma vida familiar normal é o ideal tanto para a criança com deficiência como para o restante da família. O sacrifício para o bem estar não deve ocorrer e sim oferecer a ela as mesmas necessidades das outras crianças: afeto, cuidado e oportunidades para seu progresso, autocontrole e crescimento social. ${ }^{11}$

\section{CONCLUSÃO}

Os resultados obtidos neste estudo demonstram a maior dificuldade dos pacientes com PC avaliados na área de mobilidade e, desta forma, a necessidade de maior assistência por parte do cuidador em movimentos e posturas que facilitem a locomoção e transferência.

Verificou-se, também, a necessidade de conhecer tanto as habilidades da criança como o auxílio e participação que o cuidador oferece no dia-a-dia. Desta forma o terapeuta poderá oferecer informações e orientações de manuseios e adequações funcionais. Apesar das alterações nos componentes neuromotores observados em crianças de risco, as mesmas acabam desenvolvendo estratégias motoras que lhes permitem desempenhar atividades funcionais e os cuidadores orientados pelo terapeuta podem propor facilitações que beneficiarão a função da criança, incluindo as áreas de autocuidado e função social. Encorajar a mãe a manusear e posicionar de forma adequada e fazer com que a criança participe das atividades do dia-a-dia faz com esta desenvolva da melhor forma possível suas habilidades funcionais.

\begin{abstract}
Introduction: Cerebral Palsy is a movement and posture disorder caused by events occurring during pregnancy, childbirth, the neonatal period or during the first two years of life. Its main characteristic is the body's inability to efficiently face the effects of gravity. Due to the motor alterations, the participation of the physiotherapist in the interdisciplinary team is important. Assessment of the difficulties in cerebral palsy should take place to enable a better organization of the physiotherapeutic program. Objetive: To analyze the constraints on the functional abilities and the assistance needs of children with cerebral palsy up to 18 months of age. Methods: Seven children with a medical diagnosis of cerebral palsy, with ages between 10 and 18 months, mean age corresponding to one year and two months, were assessed. The assessment occurred by means of P.E.D.I. and the gross and normative scores in the areas of functional abilities and caregiver's assistance were analyzed. Results: The mobility area was the one in which the children with cerebral palsy had more difficulties; thus, they needed more assistance in movements and postures. Conclusion: In spite of the alterations in the neuromotor components observed in risk children, the same children end up developing motor strategies that allow them to perform functional activities; for example, the situations cited in the self-care area. The caregivers, guided by the physiotherapist, can propose the means that will benefit the child's function, his/her participation in the activity and communication development.
\end{abstract}

Keywords: Cerebral palsy; infantile development; physiotherapy; early intervention; function; assessment. 


\section{REFERÊNCIAS}

1. SCHWARTZMAN, JS. Paralisia Cerebral. Temas sobre Desenvolvimento: edição especial. São Paulo: Mennon. Jul./ago.1993; 13 (3): 4-19.

2. BOBATH, Centro. XXVI curso Bobath, método de tratamento neuro-evolutivo (Apostila). São Paulo, 1997.

3. STOKES, M. Neurologia para fisioterapeutas. São Paulo: Premier; 2000.

4. IWABE C, PIOVESANAAMSG. Estudo comparativo do tono muscular na paralisia cerebral tetraparética em crianças com lesões predominantemente corticais ou subcorticais na tomografia computadorizada de crânio. Arq. Neuro-Psiquiatr. São Paulo: 2003; 61:3A.

5. MANCINI MC, et al. Comparação do desempenho de atividades funcionais em crianças com desenvolvimento normal e crianças com paralisia cerebral. Arq. NeuroPsiquiatr. São Paulo: 2002; 60: 2 B.

6. MANCINI MC, et al. Perfil do desenvolvimento neuromotor do bebê de alto risco no primeiro ano de vida. Temas sobre o desenvolvimento. São Paulo: 1992; 8: 3-8.

7. MANCINI, M.C.. Inventário de Avaliação Pediátrica de Incapacidade (P.E.D.I.), Manual da Versão Brasileira Adaptada. Minas Gerais: Editora UFMG; 2005.

8. ALLEGRETTI, A.L.C.; MANCINI, M.C.; SCHWARTZMAN, J.S.. Estudo do desempenho funcional de crianças com paralisia cerebral diparética espástica utilizando o Pediatric Evaluation of disability inventory (P.E.D.I.). Arq. Bras. Paralis. Cereb. 2004;1:35-40.

9. OLIVEIRA, M.C.; CORDANI, L.K.. Correlação entre habilidades funcionais referidas pelo cuidador e assistência fornecida a crianças com paralisia cerebral. Arq. Bras. Paralis. Cereb. 2004;1:24-29.

10. HUNSINGER, M.M.B.Y.. O bebê e a coordenação motora. São Paulo: Summus Editorial; 2a edição; 1994.

11. FINNIE, N.R.. O manuseio em casa da criança com paralisia cerebral. São Paulo: Manole; $3^{\mathrm{a}}$ edição; 2000. 